\title{
Relación entre la práctica deportiva y la autoestima en adolescentes: proyecto DADOS
}

\author{
Carlos Bou Sospedra \\ al379860@uji.es \\ María Reyes Beltrán Valls \\ vallsm@uji.es \\ Mireia Adelantado Renau \\ adelantm@uji.es \\ Vicente Javier Ayala Tajuelo \\ vayala@uji.es \\ Diego Moliner Urdiales \\ dmoliner@uji.es
}

LIFE Research Group, Universitat Jaume I, Castellón

\section{Resumen}

Introducción: La autoestima se define como los sentimientos propios y la evaluación personal de uno mismo. Diferentes estudios de intervención sugieren que el nivel de autoestima en adolescentes podría beneficiarse de una práctica deportiva regular. Sin embargo, poco se sabe sobre qué tipo de deporte y cuántas sesiones harían falta para conseguir efectos positivos sobre los niveles de autoestima. El objetivo del presente estudio fue analizar la relación entre el tipo de deporte y el número de sesiones semanales con el nivel de autoestima en adolescentes.

Materiales y método: La muestra estuvo formada por 266 adolescentes $(13,09 \pm 0,3$ años) participantes del proyecto DADOS (Deporte, Adolescencia y Salud). La práctica deportiva (tipo de deporte y sesiones semanales) fue recogida mediante cuestionario. La autoestima se evaluó empleando el nivel 3 de la versión española del cuestionario Behaviour Assessment System for Children y se estableció una puntuación por debajo de 30 como baja autoestima. Resultados: Los análisis de covarianza revelaron que las chicas que practicaban un deporte individual presentaban una mejor autoestima $(49,43$ frente a 54,35 ; $\mathrm{p}<0,05)$. Las chicas que realizaban al menos tres sesiones de entrenamiento a la semana presentaron una mejor autoestima $(49,43$ frente a 54,$25 ; p \leq 0,05)$. No se encon traron diferencias significativas entre los chicos $(p>0,05)$.

Conclusiones: La práctica de un deporte individual y la realización de al menos tres sesiones de práctica deportiva a la semana parecen estar relacionadas con 
una mejor autoestima en chicas adolescentes. Las iniciativas enfocadas al bienestar psicológico en adolescentes deberían considerar la práctica deportiva como un factor clave, teniendo en cuanta las diferencias entre sexos.

Palabras clave: autoestima, bienestar psicológico, atletas, deporte, adolescencia.

\begin{abstract}
Introduction: Self-esteem can be defined as the feelings and evaluation of one's personal self-worth. Previous intervention research suggests that levels of selfesteem could be improved by regular sport practice in adolescents. However, little is known regarding the type of sport and weekly training sessions which could improve adolescents' self-esteem. The aim of the current study was to analyse the relationship between sport practice (i.e. type of sport and weekly training sessions) with self-esteem in adolescents.

Methods: A sample of 266 adolescents $(13.09 \pm 0.3$ years) from DADOS (Deporte, Adolescencia y Salud) study was included in the analysis. Sport practice (type of sport and weekly training sessions) was reported by questionnaire. Behavior Assessment System for Children (BASC) level 3 was used to assess adolescents' self-esteem and low self-esteem was established under 30 score.

Results: Analysis of the covariance indicated better self-esteem in girls who practiced an individual sport, compared to those who did not practice sport (49.43 vs $54.35 ; p<0.05)$. Girls who practised at least three training sessions per week showed better self-esteem ( 49.43 vs $54.25 ; p \leq 0.05)$. No significant differences were found in boys $(p>0.05)$.

Conclusions: The practice of an individual sport and performing at least three training session per week seem to be related with better self-esteem in adolescent girls. Health-related programs aimed to improve psychological wellbeing in adolescents should consider sport practice as a key factor.
\end{abstract}

Keywords: self-esteem, psychological well-being, athletes, sport, adolescence

\title{
Introducción
}

La autoestima podría definirse como los sentimientos propios y la evaluación personal de uno mismo (Crocker y Major 1989). Diversos estudios previos sugieren que la autoestima se asocia positivamente con el rendimiento académico (Hyseni Duraku y Hoxha 2018), la salud mental (Mann, Hosman, Schaalma y De Vries 2004), la satisfacción personal y las relaciones interpersonales (Du, King y Chi 2017) e inversamente con el bullying (Fanti y Henrich 2014), la depresión (Masselink 2017) y la obesidad (Griffiths, Parsons y Hill 2010) en adolescentes y adultos jóvenes. No obstante, un reciente informe de la Organización Mundial de Salud indicó que alrededor del $20 \%$ de los adolescentes expermientan algún problema de salud mental, entre los que se incluye la baja autoestima (World Health Organization 2012).

La adolescencia es un periodo crítico caracterizado por múltiples cambios psicológicos, en el que se adquieren y modifican hábitos de vida, como la práctica de actividad física, con el consiguiente beneficio o riesgo para la salud (Ortega, Ruiz, Castillo y Sjöström 2008). Diversas investigaciones han examinado la relación entre la 
práctica de actividad física y la autoestima en adolescentes (Biddle y Asare 2011; Ekeland, Heian y Hagen 2005; Lubans et al. 2016). De hecho, diferentes estudios de intervención han mostrado mejorar los niveles de autoestima a través de programas que promocionan la práctica de actividad física en adolescentes (Asare 2013; Liu, Wu y Ming 2015; Smedegaard, Christiansen, Lund-Cramer, Bredahl y Skovg aard 2016).

Una de las formas más comunes de práctica de actividad física durante esta etapa es la participación en actividades deportivas organizadas (Eime, Young, Harvey, Charity y Payne 2013). Si bien estudios previos han relacionado la práctica deportiva de élite con niveles elevados de presión, estrés y burnout (Gustafsson, Sagar y Stenling 2017), existe evidencia científica que sugiere que la práctica deportiva adecuadamente programada podría influir positivamente sobre el desarrollo del bienestar psicológico en adolescentes (Fraser-Thomas, Côté y Deakin 2007). Entre estos beneficios para la salud se incluye una mejora de los niveles de autoestima, que podría estar asociada con las características específicas de dichas actividades deportivas (Esteban-Cornejo, Tejero-Gonzalez, Sallis y Veiga 2015; Pedersen y Saltin 2006; Vella, Swann, Allen, Schweickle y Magee 2017). Sin embargo, la mayor parte de la evidencia científica examina conceptos globales como el bienestar psicológico o la salud mental, sin focalizase en conceptos concretos como la autoestima. De igual modo pasa con la práctica de actividad física, ya que se valora el nivel de actividad sin tener en cuenta el tipo de disciplina deportiva o el volumen de la misma. Por ello, el objetivo del presente estudio fue analizar la relación entre la práctica deportiva (es decir, tipo de deporte y número de sesiones semanales) y el nivel de autoestima en adolescentes sanos.

\section{Método}

\section{Participantes}

Los datos del presente estudio pertenecen a la evaluación inicial del proyecto de investigación DADOS (Deporte, Adolescencia y Salud) y fueron recogidos entre los meses de febrero y mayo del 2015. El objetivo principal del proyecto DADOS es analizar la influencia que tiene la práctica deportiva sobre la salud, el rendimiento académico y el nivel de bienestar psicológico durante la etapa de la adolescencia. Un total de 266 adolescentes, con datos válidos de práctica deportiva y nivel de autoestima, fueron incluidos en los análisis. Los participantes fueron seleccionados en centros de educación secundaria y clubs deportivos y cumplían con los criterios de inclusión generales del proyecto DADOS (alumnos de $2 .^{\circ}$ de ESO, libres de enfermedades crónicas y tratamientos farmacológicos). Todos los participantes recibieron una explicación detallada del protocolo de investigación y tanto ellos como sus padres o tutores legales firmaron un consentimiento informado. El proyecto DADOS se ha desarrollado siguiendo las recomendaciones éticas de la Declaración de Helsinki 1961 (revisión de Fortaleza 2013) y su protocolo de investigación ha sido aprobado por la Comisión Deontológica de la Universitat Jaume I de Castellón.

\section{Instrumentos de evaluación}

\section{- Desarrollo madurativo}

El nivel de desarrollo madurativo fue autoreportado por los adolescentes según los 5 estadios descritos por Tanner y Whitehouse (1976). 


\section{- Estatus socioeconómico}

El estatus socioeconómico fue evaluado mediante la Family Affluence Scale (FAS) desarrollada por Currie et al. (2008). Esta escala presenta un rango de 0 a 8 puntos y se basa en las posesiones materiales de la familia, la propiedad de automóviles, el mobiliario de las habitaciones, la propiedad de ordenadores y el acceso a internet en el domicilio.

- Práctica deportiva

El tipo de deporte y el número de sesiones semanales de entrenamiento fueron autoreportados mediante cuestionario. Según el tipo de deporte, los participantes fueron clasificados en 3 categorías: deporte individual, deporte colectivo y ningún deporte. En base al número de sesiones de entrenamiento semanales, los participantes fueron dicotomizados: $<3$ sesiones semanales frente $a \geq 3$ sesiones semanales.

\section{- Autoestima}

La versión en castellano del Behaviour Assesment System for Children (BASC-3) se utilizó para medir el nivel de autoestima de los participantes (González, Fernández, Pérez y Santamaría 2004; Reynolds y Kamphaus 2004). EI BASC-3 es un instrumento internacionalmente validado para la evaluación del desarrollo socioemocional en niños y adolescentes. La autoestima está considera dentro de las escalas clínicas y el propio cuestionario establece un punto de corte según la edad, que permite clasificar a los adolescentes en baja autoestima cuando su puntuación es inferior a 30 puntos.

\section{Análisis estadístico}

Los datos se presentan como medias \pm desviación estándar o frecuencia (\%). Las diferencias entre sexos se analizaron mediante la prueba $t$ de Student en caso de variables continuas y utilizando el test chi-cuadrado en caso de variables categóricas. El tamaño del efecto, la d de Cohen, se calculó e interpretó siguiendo los valores de corte establecidos por Cohen (1988): pequeño $(\sim 0,20)$, medio $(\sim 0,5) \circ$ grande $(\sim 0,8)$. La relación entre la autoestima y los diferentes indicadores de práctica deportiva se analizaron en modelos de regresión lineal separados que siempre tuvieron el nivel madurativo y el estatus socioeconómico como covariables.

Se realizaron análisis de covarianza (ANCOVA) ajustando por nivel madurativo y estatus socioeconómico para examinar diferencias en la autoestima entre las categorias de tipo de práctica deportiva y sesiones de entrenamiento semanal. Se calculó el tamaño del efecto mediante el parámetro $\eta^{2}$. Los análisis se realizaron por separado para chicas y chicos. Todos los análisis se realizaron utilizando el software IBM SPSS V. 22.0 (Armonk, NY: IBM Corp) y se estableció un nivel de significación de $p<0,05$.

\section{Resultados}

Como se observa en la tabla 1 , el $\sim 70 \%$ de la muestra practicaba deporte y el $\sim 63 \%$ realizaba al menos 3 sesiones de práctica deportiva a la semana. Un $4 \%$ de los participantes presentó baja autoestima, con una prevalencia mayor entre las chicas ( $6 \%$ chicas frente a $1 \%$ chicos; $p<0,001)$. Los chicos mostraron una mayor autoestima que las chicas $(55,1$ frente a 51,4 , d de cohen $=0,497 ; p \leq 0,001)$. 
Tabla 1

Características de los participantes por sexo

\begin{tabular}{|c|c|c|c|c|c|}
\hline & \multirow{2}{*}{$\begin{array}{c}\text { Total } \\
\mathrm{N}=266\end{array}$} & \multirow{2}{*}{$\begin{array}{l}\text { Chicos } \\
N=140\end{array}$} & \multirow{2}{*}{$\begin{array}{l}\text { Chicas } \\
N=126\end{array}$} & \multirow[t]{2}{*}{$\mathbf{T}$} & \multirow[t]{2}{*}{$\mathbf{P}$} \\
\hline & & & & & \\
\hline Edad (años) & $13,9 \pm 0,3$ & $13,9 \pm 0,3$ & $13,8 \pm 0,3$ & 0,287 & 0,923 \\
\hline $\begin{array}{l}\text { Nivel de maduración } \\
(\mathrm{II}-\mathrm{V})(\%)\end{array}$ & $8 / 33,7 / 48,7 / 9,6$ & $10,3 / 32,4 / 43,4 / 14$ & $5,6 / 35,2 / 54,4 / 4,8$ & - & 0,351 \\
\hline $\begin{array}{l}\text { Nivel socioeconómico } \\
(0-8)(\%)\end{array}$ & $4,16 \pm 1,4$ & $4 \pm 1,3$ & $4,3 \pm 1,4$ & - & 0,859 \\
\hline \multicolumn{6}{|l|}{ Tipo de deporte } \\
\hline Ningún deporte (\%) & $81(30,5)$ & $23(16.4)$ & $58(46)$ & & \\
\hline Deporte individual (\%) & $84(31,6)$ & $38(27.1)$ & $46(36,5)$ & - & $<0,001$ \\
\hline Deporte colectivo (\%) & $101(38)$ & $79(56.4)$ & $22(17.5)$ & & \\
\hline \multicolumn{6}{|c|}{ Sesiones semanales de entrenamiento } \\
\hline >3 sesiones & $168(63,2)$ & $102(72,9)$ & $66(52,4)$ & - & 0,001 \\
\hline \multicolumn{6}{|l|}{ Autoestima } \\
\hline $\begin{array}{l}\text { Puntuación autoestima } \\
\text { Baja }^{\mathrm{a}}(\%)\end{array}$ & $\begin{array}{r}53,4 \pm 7,4 \\
10(3,8)\end{array}$ & $\begin{array}{c}55,1 \pm 4,7 \\
2(1,4)\end{array}$ & $\begin{array}{c}51,4 \pm 9,2 \\
8(6,3)\end{array}$ & $-4,068$ & $<0,001$ \\
\hline
\end{tabular}

Los datos se presentan como media \pm desviación estándar o frecuencia (\%). Las diferencias entre sexos fueron examinadas mediante el t-test y el test chi-cuadrado.

Los valores estadísticamente significativos están resaltados en negrita.

${ }^{a}$ Sujetos con una puntuación por debajo de 30 en el cuestionario BASC-3.

$\mathrm{El}$ análisis de regresión lineal mostró una asociación positiva entre el número de sesiones de entrenamiento a la semana y la autoestima en chicas $(\beta=0.977 ; p=0.022)$, pero no en chicos (véase la tabla 2).

\section{Tabla 2}

Análisis de regresión lineal entre el número de sesiones de entrenamiento a la semana y la autoestima, ajustado por nivel de maduración y nivel socioeconómico ( $n=266)$

\begin{tabular}{cll}
\cline { 2 - 3 } & $\beta$ & $\mathrm{p}$ \\
\hline Autoestima & \\
Chicos & $-0,247$ & 0,348 \\
Chicas & 0,977 & 0,022 \\
\hline
\end{tabular}

$\beta$ : coeficiente de regresión estandarizado.

Los análisis de covarianza (figura 1) revelaron que las chicas que practicaban un deporte individual presentaban una mejor autoestima que las que no practicaban deporte $\left(49,43\right.$ frente a $\left.54,35, F=3,852, \eta^{2}=0,060 ; p<0,05\right)$. No se encontraron diferencias estadísticamente significativas en el nivel de autoestima según el tipo de deporte en chicos $(p>0,05)$. 

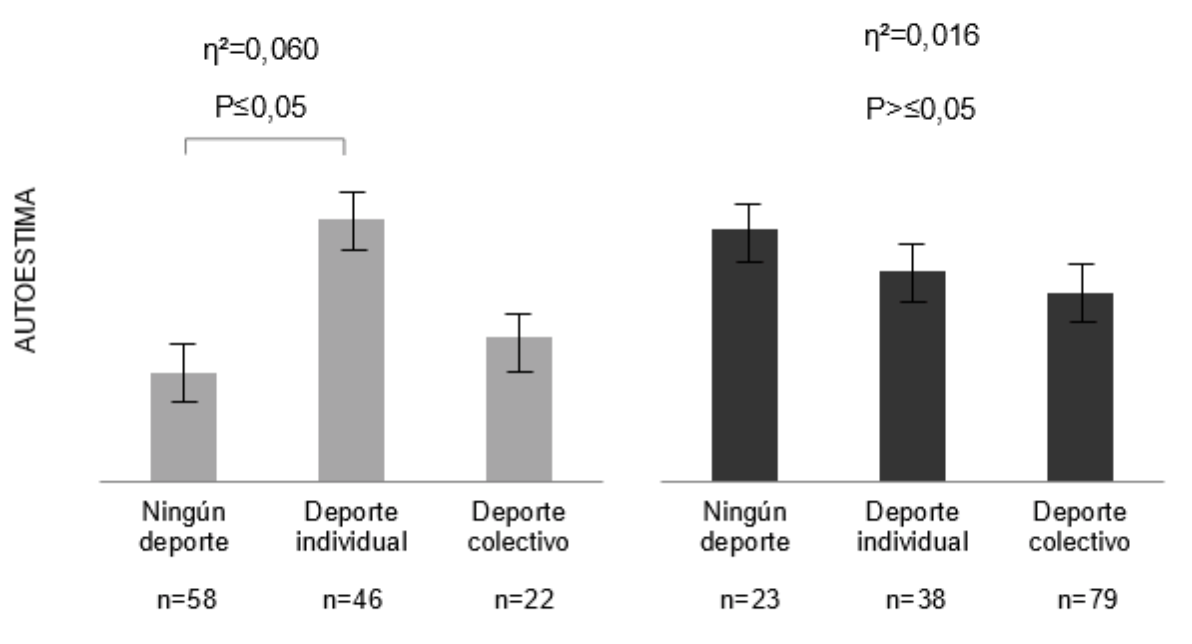

Figura 1. Diferencias en la autoestima según el tipo de práctica deportiva utilizando análisis de covariancia, la media estimada y los intervalos de confianza del $95 \%$ (barras de error) ajustando por nivel de maduración y nivel socioeconómico. $\eta^{2}$ : estadístico del análisis de covarianza.

Las chicas que realizaban al menos tres sesiones de entrenamiento a la semana (figura 2) presentaron una mejor autoestima $\left(49,43\right.$ frente a $54,25, F=5,534, \eta^{2}=0,043$; $p \leq 0,05)$. No se encontraron diferencias significativas en el nivel de autoestima según el número de sesiones de entrenamiento semanales en chicos $(p>0,05)$.
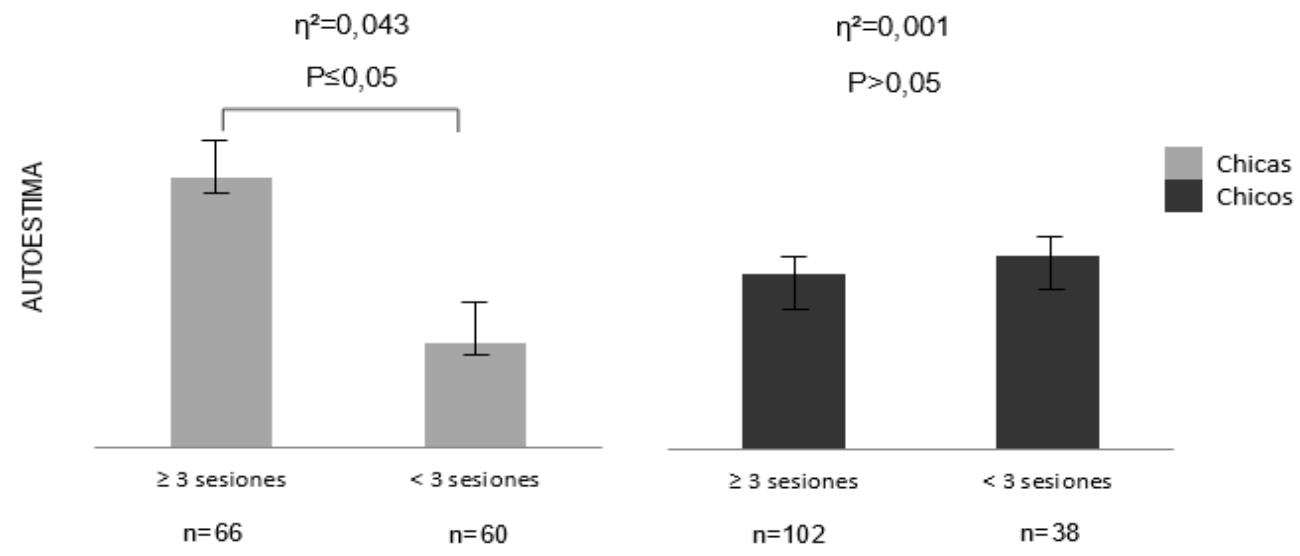

Figura 2. Diferencias en la autoestima según el número de sesiones de entrenamiento a la semana utilizando análisis de covariancia, la media estimada y los intervalos de confianza del $95 \%$ (barras de error) ajustando por nivel de maduración y nivel socioeconómico. $\eta^{2}$ : estadístico del análisis de covarianza.

\section{Discusión}

Los hallazgos principales de este estudio indican una relación positiva entre la práctica de un deporte individual y el nivel de autoestima en chicas adolescentes, comparado con aquellas que no practican deporte. Si bien la práctica deportiva no estuvo relacionada con la autoestima en chicos, la realización de al menos 3 sesiones de 
práctica deportiva semanales se asoció con un mayor nivel de autoestima en chicas. Estos resultados contribuyen al conocimiento actual sugiriendo que la práctica deportiva podría tener una influencia positiva sobre los niveles de autoestima, especialmente entre las chicas adolescentes.

Estudios similares han encontrado una relación positiva entre la práctica deportiva y la autoestima en adolescentes (Balaguer, Atienza y Duda 2012; Fabio y Towey 2018; Findlay y Bowker 2009; Medrano, Mateos y Martos 2017; von Rosen, Olofsson, Väsbom y Heijne 2018; Wagnsson, Lindwall y Gustafsson 2014). Por ejemplo, Balaguer, Atienza y Duda (2012) examinaron una muestra de 917 adolescentes españoles y encontraron una asociación positiva entre la frecuencia de práctica deportiva y la autopercepción. De forma similar, von Rosen, Olofsson, Väsbom y Heijne (2018) mostraron que los adolescentes que entrenaban una media de 7-8 horas semanales presentaban mayores niveles de autoestima que aquellos que entrenaban menos de 4 horas semanales. De igual modo, Fabio y Towey (2018) determinaron que los deportistas que practicaban artes marciales (deporte individual) presentaban mejores niveles de autoestima que aquellos que practicaban deportes colectivos. Además, Medrano, Meteos y Martos (2017) asociaron mejores niveles de autoconfianza, directamente relacionada con la autoestima, a la práctica de deportes individuales y del fútbol. Sin embargo, de forma contraria a nuestros resultados, estudios previos identificaron una relación positiva entre la práctica de un deporte colectivo y la autoestima (Slutzky y Simpkins 2009; von Rosen, Olofsson, Väsbom y Heijne 2018).

La relación positiva encontrada en nuestro estudio entre la práctica de un deporte individual y la autoestima en adolescentes podría deberse al hecho de que el tiempo dedicado a las actividades deportivas individuales puede ofrecer a los participantes una información más clara sobre sus capacidades y estándares de rendimiento, dada la importancia del desempeño individual en la consecución de los resultados deportivos. Además es más probable que los practicantes de un deporte individual atribuyan el rendimiento de la práctica a sus habilidades en lugar de a las habilidades de sus compañeros (Pedersen y Seidman 2004; Slutzky y Simpkins 2009), lo que podría aumentar su autoestima.

Respecto a la diferencia hallada entre géneros, la literatura muestra una brecha significativa, de modo que los chicos tienden a reportar niveles más altos de autoestima que las chicas durante la adolescencia (Bleidorn et al. 2016; Orth y Robins 2014; Orth, Trzesniewski y Robins 2010). De igual modo, las atletas son más propensas a experimentar miedo al fracaso, lo que podría conllevar una baja autoestima en situaciones donde sus fallos repercutan en los demás, como por ejemplo en la práctica de deportes colectivos (Correira, Rosado, Serpa y Ferreira 2017). Además, un $32 \%$ de las adolescentes españolas presentan bajos niveles de práctica deportiva frente a un $17 \%$ de los chicos (World Health Organization 2018), por lo que el efecto del número de sesiones de entrenamiento semanal sobre la autoestima podría ser más determinante en chicas.

\section{Limitaciones y puntos fuertes}

Los resultados deben ser interpretados con cautela debido al diseño transversal del estudio, que no permite establecer relación causa-efecto. Además, los análisis no consideraron el efecto de otras variables que podrían influir en la relación entre práctica deportiva y niveles de autoestima (por ejemplo, composición corporal o nivel socioeconómico). No obstante, nuestro estudio presenta diversas fortalezas entre las cuales cabe destacar la homogeneidad de la muestra en relación a su edad, la 
utilización de un cuestionario validado para la evaluación de la autoestima y la inclusión de variables con gran influencia en la relación estudiada, como el nivel del desarrollo madurativo.

\section{Conclusión}

La práctica de un deporte individual y la realización de al menos tres sesiones de práctica deportiva a la semana parecen estar relacionadas con una mejor autoestima en chicas adolescentes. Dado el gran número de beneficios que la práctica deportiva presenta sobre el bienestar psicológico, especialmente la práctica de deportes individuales, las familias y las instituciones de salud pública y educativas deberían tener en cuenta su promoción entre los adolescentes, así como considerar las diferencias inherentes al sexo a la hora de promover iniciativas de promoción de actividad física. Se requieren futuros estudios que clarifiquen las características específicas de la práctica deportiva que reportan beneficios sobre el bienestar psicológico con el fin de implementar programas de promoción de la salud más específicos y eficaces.

\section{Referencias bibliográficas}

Asare, Mavis. 2013. Physical activity, sedentary behaviour and mental health in young people. London: Loughborough University.

Balaguer, Isabel, Francisco L. Atienza y Joan L. Duda. 2012. "Self-Perceptions, SelfWorth and Sport Participation in Adolescents». The Spanish Journal of Psychology, 15(2): 624-630.

Biddle, Stuart J. H. y Mavis Asare. 2011. "Physical activity and mental health in children and adolescents: A review of reviews». British Journal of Sports Medicine, 45(11): 886-895. https://doi.org/10.1136/bjsports-2011-090185

Bleidorn, Wiebke, Ruben C. Arslan, Jaap J.A. Denissen, Peter J. Rentfrow, Jochen E. Gebauer, Jeff Potter y Samuel D. Gosling. 2016. «Age and gender differences in self-esteem: A cross-cultural window». Journal of Personality and Social Psychology, 111(3): 396-410. https://doi.org/10.1037/pspp0000078

Cohen, Jacob. 1988. Statistical Power Analysis for the Behavioral Sciences. New York: Routledge. https://doi.org/10.4324/9780203771587

Correira, Marco, Antonio Rosado, Sdónio O. Serpa y Vítor Ferreira. 2017. «Fear of failure in athletes: gender, age and type of spor difference». Revista Iberoamericana de Psicología Del Ejercicio y El Deporte, 12: 185-193.

Crocker, Jennifer y Brenda Major. 1989. «Social Stigma and Self-Esteem». Psychological Science, 96(4): 608-630.

Currie, Candace, Michael Molcho, William Boyce, Bjørn Holstein, Torbjørn Torsheim y Matthias Richter. 2008. «Researching health inequalities in adolescents: The development of the Health Behaviour in School-Aged Children (HBSC) Family Affluence Scale». Social Science and Medicine, 66(6): 1429-1436. https://doi.org/10.1016/j.socscimed.2007.11.024

Du, Hongfei, Ronnel B. King y Peilian Chi. 2017. "Self-esteem and subjective wellbeing revisited: The roles of personal, relational, and collective self-esteem». PLOS ONE, 12(8): 1-17.

Eime, Rochelle M., Janet A. Young, Jack T. Harvey, Melanie J. Charity y Waren R. Payne. 2013. «A systematic review of the psychological and social benefits of participation in sport for adults: Informing development of a conceptual model of health through sport». International Journal of Behavioral Nutrition and Physical Activity, 10(1): 1. https://doi.org/10.1186/1479-5868-10-135 
Ekeland, Eilin, Frode Heian y Kåre B. Hagen. 2005. «Can exercise improve self esteem in children and young people\%? A systematic review of randomised controlled trials». British Journal of Sports Medicine, 39: 792-798. https://doi.org/10.1136/bjsm.2004.017707

Esteban-Cornejo, Irene, Carlos M. Tejero-Gonzalez, James F. Sallis y Oscar L. Veiga. 2015. "Physical activity and cognition in adolescents: A systematic review». Journal of Science and Medicine in Sport, 18(5): 534-539. https://doi.org/10.1016/j.jsams.2014.07.007

Fabio, Rosa A. y Giula E. Towey. 2018. "Cognitive and personality factors in the regular practice of martial arts». The Journal of Sports Medicine and Physical Fitness, 58(6): 933-943. https://doi.org/10.23736/S0022-4707.17.07245-0

Fanti, Kostas y Christopher C. Henrich. 2014. «Effects of Self-esteem and Narcissim on Bullying and Victimization during Early Adolescence». The Journal of Early Adolescence, 35(1): 5-29. https://doi.org/10.1177/0272431613519498

Findlay, Leanne C. y Anne Bowker. 2009. "The link between competitive sport participation and self-concept in early adolescence: A consideration of gender and sport orientation». Journal of Youth and Adolescence, 38(1): 29-40. https://doi.org/10.1007/s10964-007-9244-9

Fraser-Thomas, Jessica L., Jean Côté y Janice Deakin. 2007. «Youth sport programs: an avenue to foster positive youth development». Physical Education and Sport Pedagogy, 10(2005): 19-40. https://doi.org/10.1080/1740898042000334890

González, Javier, Silvia Fernández, Emili Pérez y Pablo Santamaría. 2004. Adaptación española del sistema de evaluación de la cnducta en niños y adolescentes: BASC. Madrid: TEA.

Griffiths, Lucy J., Tessa J. Parsons y Andrew J. Hill. 2010. «Self-esteem and quality of life in obese children and adolescents: A systemati review». International Journal of Pediatric Obesity, 5: 282-304. https://doi.org/10.3109/17477160903473697

Gustafsson, Henrik, Siddharth S. Sagar y Andreas Stenling. 2017. «Fear of failure, psychological stress, and burnout among adolescent athletes competing in high level sport». Scandinavian Journal of Medicine and Science in Sports, 27(12): 2091-2102. https://doi.org/10.1111/sms.12797

Hyseni Duraku, Zamira y Linda Hoxha. 2018. "Self-esteem, study skills, self-concept, social support, psychological distress, and coping mechanism effects on test anxiety and academic performance». Health Psychology Open, 5(2). https://doi.org/10.1177/2055102918799963

Liu, Mingli, Lang Wu y Qingsen Ming. 2015. «How does physical activity intervention improve self-esteem and self-concept in children and adolescents? Evidence from a meta-analysis». PLOS ONE, 10(8): 1-17. https://doi.org/10.1371/journal.pone.0134804

Lubans, David, Justin Richards, Charles Hillman, Guy Faulkner, Mark Beauchamp, Michael Nilsson, Paul Kelly, Jordan Smith, Lauren Raine y Stuart Biddle. 2016. «Physical Activity for Cognitive and Mental Health in Youth: A Systematic Review of Mechanisms». Pediatrics, 138(3): e20161642-e20161642. https://doi.org/10.1542/peds.2016-1642

Mann, Michelle, Clemens M. H. Hosman, Herman P. Schaalma y Nanne K. De Vries. 2004. «Self-esteem in a broad-spectrum approach for mental health promotion». Health Education Research, 19(4): 357-372. https://doi.org/10.1093/her/cyg041

Masselink, Maurits. 2017. "Self-esteem in Early Adolescence as Predictor of Depressive Symptoms in Late Adolescence and Early Adulthood\%: The Mediating Role of Motivational and Social Factors». Journal of Youth and Adolescence, 47(5): 932-946. https://doi.org/10.1007/s10964-017-0727-z 
Medrano, Enrique F., María E. Mateos y Rocio G. Martos. 2017. «Confidence in Sport in School Age». Revista Iberoamericana de Diagnóstico y Evaluación, 2(34): 158-171. https://doi.org/10.21865/RIDEP44.2.13

Ortega, Francisco B., Jonatan R. Ruiz, Manuel J. Castillo y Michael Sjöström. 2008. «Physical fitness in childhood and adolescence: A powerful marker of health». International Journal of Obesity, 32(1): 1-11. https://doi.org/10.1038/sj.ijo.0803774

Orth, Ulrich y Richard W. Robins. 2014. «The Development of Self-Esteem». Current Directions in Psychological Science, 23(5): 381-387. https://doi.org/10.1177/0963721414547414

Orth, Ulrich, Kali H. Trzesniewski y Richard Robins. 2010. «Self-Esteem Development From Young Adulthood to Old Age: A Cohort-Sequential Longitudinal Study». Journal of Personality and Social Psychology, 98(4): 645-658. https://doi.org/10.1037/a0018769

Pedersen, Bente K. y Bengt Saltin. 2006. «Bidirectional associations between sport involvement and mental health in adolescence». Medicine and Science in Sports and Exercise, 18(5): 3-63. https://doi.org/10.1111/sms.12581

Pedersen, Sara y Edward Seidman. 2004. "Team sports achievement and self-esteem development among urban adolescent girls». Psychology of Women Quarterly, 28(4): 412-422. https://doi.org/10.1111/j.1471-6402.2004.00158.x

Reynolds, Cecil R. y Randy W. Kamphaus. 2004. Behavior assessment system for children. Circle Pines, MN: American Guidance Service.

Slutzky, Carl B. y Sandra D. Simpkins. 2009. "The link between children's sport participation and self-esteem: Exploring the mediating role of sport selfconcept». Psychology of Sport and Exercise, 10(3): 381-389. https://doi.org/10.1016/j.psychsport.2008.09.006

Smedegaard, Søren, Lars B. Christiansen, Pernille Lund-Cramer, Thomas Bredahl y Thomas Skovgaard. 2016. «Improving the well-being of children and youths: a randomized multicomponent, school-based, physical activity intervention». BMC Public Health, 16(1): 1-11. https://doi.org/10.1186/s12889-016-3794-2

Tanner, James M. y Reg H. Whitehouse. 1976. "Clinical longitudinal standards for height, weight, height velocity, weight velocity, and stages of puberty». Arch Dis Child, 51(3): 170-179.

Vella, Steward A., Christian Swann, Mark S. Allen, Matthew J. Schweickle y Christopher Magee. 2017. «Bidirectional associations between sport involvement and mental health in adolescence». Medicine and Science in Sports and Exercise, 49(4): 687-694. https://doi.org/10.1249/MSS.0000000000001142

von Rosen, Philip, Oskar Olofsson, Sebastian Väsbom y Annette Heijne. 2018. "Correlates of health in adolescent elite athletes and adolescents: A crosssectional study of 1016 adolescents». European Journal of Sport Science, 0(0): 1-10. https://doi.org/10.1080/17461391.2018.1552721

Wagnsson, Stefan, Magnus Lindwall y Henrik Gustafsson. 2014. «Participation in Organized Sport and Self-Esteem Across Adolescence: The Mediating Role of Perceived Sport Competence». Jorunal of Sport \& Exercise Psychology, 36: 584-594.

World Health Organization. 2012. Adolescent mental health: Mapping actions of nongovernmental organizations and other international development organizations. Geneva: World Health Organization.

World Health Organization. 2018. Spain: Physical activity factsheet. Health Topics. Recuperado de:

https://www.cdc.gov/healthyplaces/healthtopics/physactivity.htm 